\title{
Superdense dark matter clumps
}

\author{
V.I. Dokuchaev* \\ Institute for Nuclear Research of the Russian Academy of Sciences \\ E-mail: dokuchaevelngs.infn.it
}

\section{V.S. Berezinsky}

Laboratori Nazionali del Gran Sasso; Center for Astroparticle Physics at LNGS

\section{Yu.N. Eroshenko}

Institute for Nuclear Research of the Russian Academy of Sciences

E-mail: eroshenko@ms2.inr.ac.ru

\section{Kachelrieß}

Institutt for fysikk, NTNU Trondheim, Trondheim, Norway

\section{Aa. Solberg}

Institutt for fysikk, NTNU Trondheim, Trondheim, Norway

\begin{abstract}
We describe the cosmological scenario for the formation of superdense dark matter clumps. As an interesting particular example the case of superheavy neutralino as DM particles is considered. The small-scale superdense clumps form from a non-standard spiky spectrum of perturbations during the radiation dominated era. These clumps are not destroyed by tidal interactions and can be extremely dense. Superdense clumps can be observed by the gamma-radiation from dark matter annihilations and by the gravitational wave detectors, while the overproduction of primordial black holes constrains this scenario.
\end{abstract}

Identification of Dark Matter 2010-IDM2010

July 26-30, 2010

Montpellier France

\footnotetext{
*Speaker.
} 


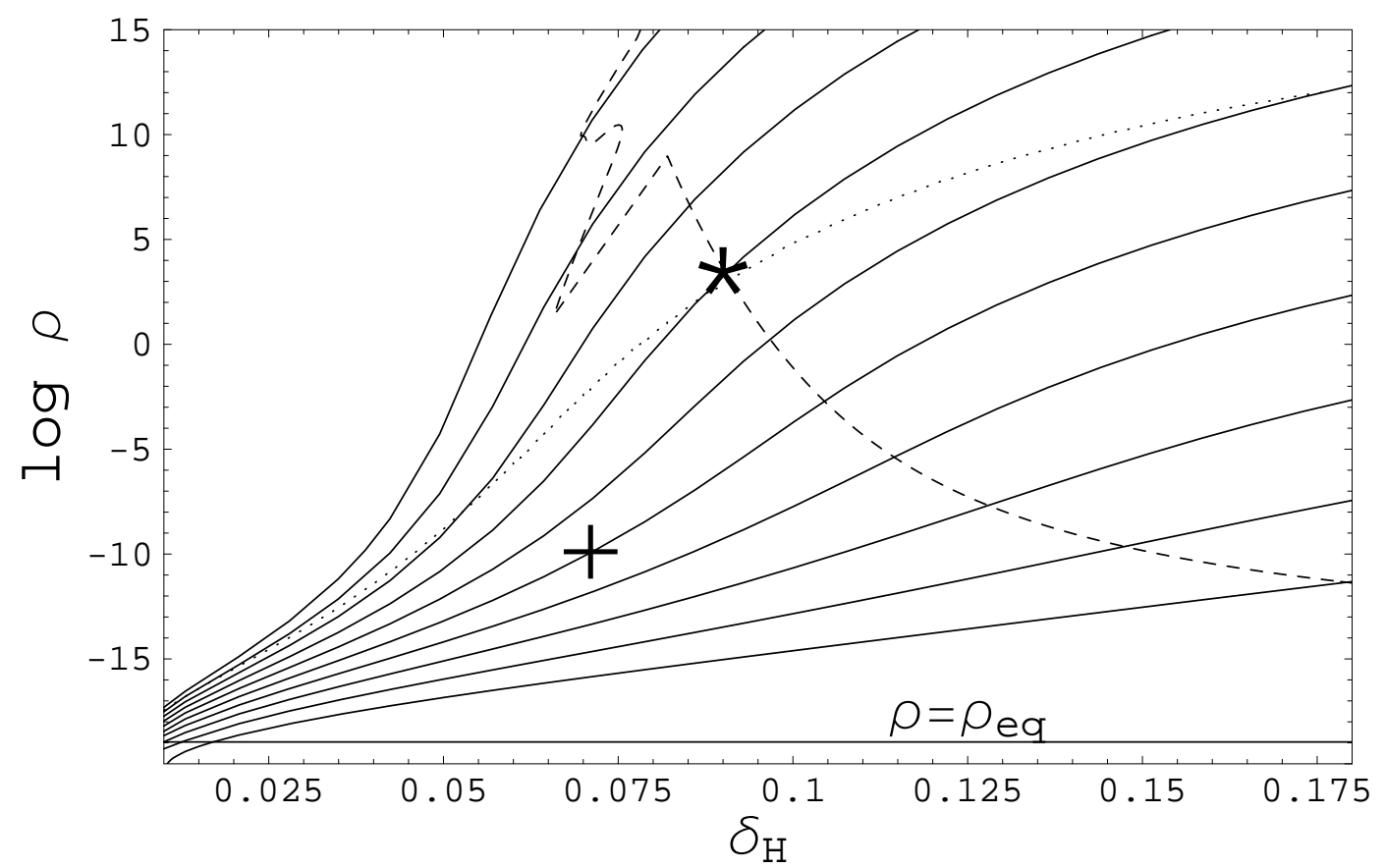

Figure 1: The mean density $\rho$ (in $\mathrm{g} \mathrm{cm}^{-3}$ ) of DM clumps as function of the perturbation $\delta_{\mathrm{H}}$ in the radiation density on the horizon scale; solid lines from top to bottom are for for clump masses $M=10^{-10}, 10^{-5}, \ldots$, $10^{35} \mathrm{~g}$. The dashed line is the bound on the clump density from primordial black holes overproduction with threshold $\delta_{c}=0.7$. The time of two-body gravitational relaxation inside the clump cores is less then the age of the Universe for clumps above the dotted lines for DM particles masses $m_{\chi}=10^{11} \mathrm{GeV}$. The star marks favourable parameters for annihilations, and the cross marks a typical example considered for comparison.

Superheavy particles can be produced at the end of inflation and they can play the role of DM particles $[1,2]$. Gravitational production in the nonstationary gravitational field provides the natural mechanism for the origin of superheavy dark matter [3]. We shall use as candidate for superheavy DM particles the neutralino with masses $10^{11} \mathrm{GeV}$ in the model of superheavy supersymmetry, as suggested in [4].

The possibility of indirect detection of stable superheavy dark matter particles depends on their annihilation rate, that scales roughly as $\dot{N}_{\text {ann }} \propto m_{\chi}^{-4}$. Since backgrounds like cosmic rays from astrophysical sources or the diffuse photon flux decrease only as $1 / E^{\alpha}$ with $\alpha \leq 3$, indirect detection of DM seems to become more and more difficult for increasing DM masses. The possibility which overcomes this difficulty is the annihilation in the superdense central region of DM clumps [5], but one needs the realistic scenario for the very high density of DM. The 2nd possibility is the formation of superdense clumps [6], [7] with very high mean density.

The mass spectrum of DM clumps has a low-mass cutoff $M_{\min }$ due to the leakage of particles from a clump. This mass is strongly model dependent. The mass spectrum of DM clumps formed by standard $\sim 100 \mathrm{GeV}$ neutralinos has a cutoff near the Earth mass. The cutoff can be diminished significantly in the case of superheavy particles. For a $10^{11} \mathrm{GeV}$ bino, this mass is only 34 times greater than the particle mass. In the case of bino the free streaming mass defines the 2 nd cutoff. 
In the case of a higgsino, the free-streaming mass is negligibly small, and free-streaming plays no role for the evolutions of perturbations.

The clumps can be produced effectively at RD stage only from non-standard spectra containing spikes. A sharp peak emerges in the fluctuation spectrum if an inflationary potential $V(\phi)$ has a flat segment. A peak emerges in the perturbation spectrum on the corresponding scale. The spectrum outside the peak can have an ordinary shape. In particular, it can be approximately HarrisonZel'dovich spectrum, and can give rise to galaxies, clusters and superclusters according to the standard scenario. Dark matter clumps are formed in a wide range of masses, if the spectrum of primordial density perturbations has a power-law form. If on the contrary the spectrum has a peak on some scale, then clumps are formed mostly in a narrow range of masses, near the peak.

The formation of clumps from entropy perturbations was considered in [6]. We deneralize that method for the evolution of adiabatic perturbations during the radiation dominated epoch. For adiabatic perturbations the initial velocity of DM shell is non-zero and is defined by the known analytic solution for linear stage. Within this formalism, we found the density of the clump $\rho=$ $\rho\left(M, \delta_{\mathrm{H}}\right)$ as function of its mass $M$ and the radiation perturbation value on the horizon scale $\delta_{\mathrm{H}}$, see Fig. 1. The details of the calculations can be found in [8].

Restriction on the spectrum of the adiabatic perturbations comes from limits on primordial black holes, which can form from the same spectrum of perturbation. In the case of entropy perturbations PBHs do not form. The corresponding restrictions are shown in Fig. 1 by the dashed curve. The local minimum on the curve corresponds to the Hawking evaporation of PBHs. The allowed region of parameters is under the dashed curve. PBHs with masses $\leq 10^{9} \mathrm{~g}$ are not constrained by Big Bang Nucleosynthesis effects, because they are evaporated very early. In the allowed region the PBHs are constrained only by the entropy production [9].

The first stage of clumps evolution is the ordinary gravitational contraction. Other processes can become important at the second stage: (i) two-body gravitational scattering and (ii) some limiting effect like Fermi degeneracy or the intensive annihilation of particles. How can it be that the gravitational two-body scattering becomes the dominant process for elementary particles? It occurs for the superheavy particles because the gravitational scattering is proportional to $\mathrm{m}^{2}$, while EW scattering of these particles is inversely proportional to $\mathrm{m}^{2}$.

In the central parts (cores) of superdense clumps the two-body gravitational scattering of superheavy DM particles may be the dominant process. This results in the "gravithermal catastrophe" - a well known effect in the dynamical evolution of dense stellar systems. This process takes place if the gravitational relaxation time is shorter than the Universe age. In this regime the evaporation of particles from the core becomes the dominant process, responsible for the evolution of the clumps. For clumps above the dotted lines in the Figure 1, relaxation results in the "gravithermal catastrophe" producing an isothermal profile $\propto r^{-2}$ with a tiny new core. This effect is important for the annihilation of superheavy particles, because the annihilation signals are too weak for observations without taking into account the "gravithermal catastrophe".

Do any physical processes exist that prevent the extremely large densities in the clump center? The first candidate for such process is given by the Electroweak elastic scattering of particles or self-interaction. Another effect is the particle annihilation. This effect was studied in [10], [11]. If superheavy DM particles are fermions like in the case of neutralinos, there is quite different effect which stops the core contraction at much larger radius. This effect is the pressure of Fermi 
degenerated gas.

Superdense clumps cannot be composed of standard $100 \mathrm{GeV}$ neutralinos, since their annihilations would overproduce the diffuse gamma radiation. Let us consider the superheavy neutralino. We calculate the annihilation rate in a single clump and the resulting flux for different types of particles and compare the result with the observational limits. In particular, the ultra-high-energy and atmospheric (secondary) neutrino observation data are used. The atmospheric neutrinos are generated in the upper atmosphere due to interactions of gamma-photons, nucleons and leptons with atoms. We include all possible channels into calculations. In the case of a higgsino, the annihilation signal is additionally enhanced by the Sommerfeld effect. For the optimistic parameters of clumps and for a superheavy bino as DM particle, the flux is several orders higher in comparison with the experimental upper limits. For the pessimistic choice of parameters the flux is several orders lower. Therefore the annihilation rate of stable superheavy neutralinos may be large enough to be detectable, if primordial density perturbations are spiky.

It has been already suggested that interferometric detectors for gravitational waves like LISA have the capability to detect the tiny variation of the gravitational field, when a compact object passes near the detector. Superdense clumps may be included into this list. The observable signal is caused by the gravitational tidal force which changes the interferometer arm length and produces correspondingly a phase shift.

This work was supported by the grant of the Leading scientific school 3517.2010.2.

\section{References}

[1] V. Berezinsky, M. Kachelrieß and A. Vilenkin, Phys. Rev. Lett. 79, 4302 (1997).

[2] V.A. Kuzmin and V.A. Rubakov, Phys. Atom. Nucl. 61, 1028 (1998) [Yad. Fiz. 61, 1122 (1998)].

[3] D.J.H. Chung, E.W. Kolb and A. Riotto, Phys. Rev. D 59, 023501 (1999); V. Kuzmin and I. Tkachev, JETP Lett. 68, 271 (1998); see also D.H. Lyth and D. Roberts, Phys. Rev. D 57, 7120 (1998).

[4] V. Berezinsky, M. Kachelrieß and M. A. Solberg, Phys. Rev. D 78, 123535 (2008).

[5] P. Blasi, R. Dick, E.W. Kolb, Astropart. Phys. 18, 57 (2002).

[6] E.W. Kolb and I.I. Tkachev, Phys. Rev. D 50, 769 (1994).

[7] P. Scott and S. Sivertsson, Phys. Rev. Lett. 103, 211301 (2009).

[8] V. Berezinsky, V. Dokuchaev, Yu. Eroshenko, M. Kachelrieß and M. A. Solberg, Phys. Rev. D 81, 103529 (2010); Phys. Rev. D 81, 103530 (2010).

[9] B.J. Carr et al., Phys.Rev. D 81, 104019 (2010); arXiv:0912.5297v2 [astro-ph.CO].

[10] V.S. Berezinsky, A.V. Gurevich and K.P. Zybin, Phys. Lett. B 294, 221 (1992).

[11] V. Berezinsky, A. Bottino and G. Mignola, Phys. Lett. B 391, 355 (1997). 